\title{
DUAL SUPERCONDUCTIVITY, EFFECTIVE STRING THEORY, AND REGGE TRAJECTORIES
}

\author{
M. BAKER and R. STEINKE \\ University of Washington \\ P.O. Box 351560, Seattle, WA 98195, USA
}

\begin{abstract}
We show how an effective field theory of long distance QCD, describing a dual superconductor, can be expressed as an effective string theory of superconducting vortices. We evaluate the semiclassical expansion of this effective string theory about a classical rotating string solution in any spacetime dimension $D$. We show that, after renormalization, the zero point energy of the string fluctuations remains finite when the masses of the quarks on the ends of the string approach zero. For $D=26$ the semiclassical energy spectrum of the rotating string formally coincides with that of the open string in classical Bosonic string theory. However, its physical origin is different. It is a semiclassical spectrum of an effective string theory valid only for large values of the angular momentum. For $D=4$ the first semiclassical correction adds the constant $1 / 12$ to the classical Regge formula for the angular momentum of mesons on the leading Regge trajectory. The excited vibrational modes of the rotating string give rise to daughter Regge trajectories determining the spectrum of hybrid mesons.
\end{abstract}

\section{The Dual Superconductor Mechanism of Confinement}

In the dual superconductor mechanism of confinement a dual Meissner effect [1, 2, 3] confines color electric flux to narrow tubes connecting a quark-antiquark pair. The dual Abelian Higgs model, which couples dual potentials $C_{\mu}$ to a monopole Higgs field $\phi$, provides an example of this mechanism. [4] The action $S\left[C_{\mu}, \phi\right]$ of the theory is

$S\left[C_{\mu}, \phi\right]=\int d^{4} x\left[-\frac{\left(\partial_{\mu} C_{\nu}-\partial_{\nu} C_{\mu}\right)^{2}}{4}-\frac{\left|\left(\partial_{\mu}-i g C_{\mu}\right) \phi\right|^{2}}{2}-\frac{\lambda}{4}\left(|\phi|^{2}-\phi_{0}^{2}\right)^{2}\right]$.

The dual coupling constant is $g=2 \pi / e$, where $e$ is the Yang-Mills coupling constant. In the confined phase the monopole fields $\phi$ condense to the value $\phi_{0}$. The dual potentials $C_{\mu}$ acquire a mass $M=g \phi_{0}$ via a dual Higgs mechanism . Quarks couple to dual potentials via a Dirac string connecting the quark-antiquark pair along a line L, the ends of which are sources and 
sinks of color electric flux. The color field of the pair destroys the dual Meissner effect near $\mathrm{L}$ so that $\phi$ vanishes on L. At distances greater than $1 / M$ the monopole field returns to its bulk value $\phi_{0}$ so that the color field is confined to a tube of radius $a=1 / M$ surrounding the line $\mathrm{L}$, which is the location of the vortex. [5, 6]

This model provides an effective theory of long distance QCD, i.e. for distances greater than $a$. Here we want to use this theory to obtain the energy levels of mesons having large angular momentum. Under such circumstances the distance between quarks is much larger than the flux tube radius and we must take into account the contribution of flux tube fluctuations to the interaction between the quark and antiquark, The fluctuating vortex line $\mathrm{L}$ sweeps out a world sheet $\tilde{x}^{\mu}$ whose boundary is the loop $\Gamma$ formed from the world lines of the moving quark-antiquark pair. Their interaction is determined by the Wilson loop $W[\Gamma]$ :

$$
W[\Gamma]=\int \mathcal{D} C_{\mu} \mathcal{D} \phi \mathcal{D} \phi^{*} e^{i S\left[C_{\mu}, \phi\right]} .
$$

The path integral (2) goes over all field configurations for which the monopole field $\phi(x)$ vanishes on some sheet $\tilde{x}^{\mu}$ bounded by the loop $\Gamma$.

\section{The Effective String Theory}

The Wilson loop $W[\Gamma]$ describes the quantum fluctuations of a field theory having classical vortex solutions. We want to express the functional integration over fields as a path integral over the vortex sheets $\tilde{x}^{\mu}$ to obtain an effective string theory of these vortices. To do this we carry out the functional integration (2) in two stages:

1. We integrate over all field configurations in which the vortex is located on a particular surface $\tilde{x}^{\mu}$, where $\phi\left(\tilde{x}^{\mu}\right)=0$. This integration determines the action $S_{\text {eff }}\left[\tilde{x}^{\mu}\right]$ of the effective string theory.

2. We integrate over all vortex sheets $\tilde{x}^{\mu}(\xi), \xi=\xi^{a}, a=1,2$. This integration gives $W[\Gamma]$ the form of an effective string theory of vortices.

The action $S_{\text {eff }}\left[\tilde{x}^{\mu}\right]$ is invariant under reparameterizations $\xi \rightarrow \xi^{\prime}(\xi)$ of the world sheet $\tilde{x}^{\mu}(\xi)$ of the vortex. We choose a particular parameterization of $\tilde{x}^{\mu}$ in terms of the amplitudes $f^{1}(\xi)$ and $f^{2}(\xi)$, of the two transverse fluctuations of the vortex.

$$
\tilde{x}^{\mu}(\xi)=x^{\mu}\left(f^{1}(\xi), f^{2}(\xi), \xi^{1}, \xi^{2}\right) .
$$

Using the parameterization (3), we can write the integration over vortex sheets as a path integral over the transverse vortex fluctuations $f^{1}(\xi)$ and 
$f^{2}(\xi)$. The path integral (2) over fields then takes the form: [7]

$$
W[\Gamma]=\int \mathcal{D} f^{1} \mathcal{D} f^{2} \Delta_{F P} e^{i S_{\mathrm{eff}}\left[\tilde{x}^{\mu}\right]},
$$

where

$$
\Delta_{F P}=\operatorname{Det}\left[\frac{\epsilon_{\mu \nu \alpha \beta}}{\sqrt{-g}} \frac{\partial x^{\mu}}{\partial f^{1}} \frac{\partial x^{\nu}}{\partial f^{2}} \frac{\partial \tilde{x}^{\alpha}}{\partial \xi^{1}} \frac{\partial \tilde{x}^{\beta}}{\partial \xi^{2}}\right]
$$

is a Faddeev-Popov determinant, and $\sqrt{-g}$ is the square root of the determinant of the induced metric $g_{a b}$,

$$
g_{a b}=\frac{\partial \tilde{x}^{\mu}}{\partial \xi^{a}} \frac{\partial \tilde{x}_{\mu}}{\partial \xi^{b}}
$$

The path integral (4) goes over fluctuations in the shape of the vortex sheet with wave lengths greater than the radius $1 / M$ of the flux tube. All the long distance fluctuations in $W[\Gamma]$ are contained in this path integral over string fluctuations.

The presence of the determinant $\Delta_{F P}$ makes the path integral (4) invariant under reparameterizations $\tilde{x}^{\mu}(\xi) \rightarrow \tilde{x}^{\mu}\left(\xi\left(\xi^{\prime}\right)\right)$ of the vortex worldsheet. Specifying the parameterization (3) is analogous to fixing a gauge in a gauge theory, and gives rise to the path integral (4) over $f^{1}(\xi)$ and $f^{2}(\xi)$ .The factor $\Delta_{F P}$ arises from writing the original field theory path integral (2) as a ratio of path integrals of two string theories [8], and reflects the field theory origin of the effective string theory . This ratio is anomaly free. Anomalies [9] present in string theory are not present in field theory.

\section{The Action of the Effective String Theory}

The parameterization invariant measure in the path integral (位) is universal and is independent of the explicit form of the underlying field theory. On the other hand, the action $S_{\text {eff }}\left[\tilde{x}^{\mu}\right]$ of the effective string theory is not universal. It is determined by the integration over all field configurations which contain a vortex on the surface $\tilde{x}^{\mu}$. However, for wavelengths $\lambda$ of the string fluctuations greater than the flux tube radius $a$, which are those included in (蛋), the action $S_{\text {eff }}\left[\tilde{x}^{\mu}\right]$ can be expanded in powers of the extrinsic curvature tensor $\mathcal{K}_{a b}^{A}$ of the sheet $\tilde{x}^{\mu}$,

$$
S_{\mathrm{eff}}\left[\tilde{x}^{\mu}\right]=-\int d^{4} x \sqrt{-g}\left[\sigma+\beta\left(\mathcal{K}_{a b}^{A}\right)^{2}+\ldots\right] .
$$

The extrinsic curvature tensor is

$$
\mathcal{K}_{a b}^{A}=n_{\mu}^{A}(\xi) \frac{\partial^{2} \tilde{x}^{\mu}}{\partial \xi^{a} \partial \xi^{b}},
$$


where $n_{\mu}^{A}(\xi), A=1,2$ are vectors normal to the worldsheet at the point $\tilde{x}^{\mu}(\xi)$. The string tension $\sigma$ and the rigidity $\beta$ are determined by the parameters of the underlying effective field theory, whose long distance fluctuations are described by the effective string theory .

The expansion parameter in (7) is the ratio $(a / \lambda)^{2}$ of the square of the flux tube radius to the square of the wave length of the string fluctuations. In mesons of angular momentum $J$ this parameter is of order $1 / J$. For mesons of large angular momentum the effective action (7) can therefore be approximated by the Nambu-Goto action,

$$
S_{\text {eff }}=S_{\mathrm{NG}} \equiv-\sigma \int d^{4} x \sqrt{-g},
$$

and the path integral for $W[\Gamma]$ becomes

$$
W[\Gamma]=\int \mathcal{D} f^{1} \mathcal{D} f^{2} \Delta_{F P} e^{i \sigma \int d^{4} x \sqrt{-g}} .
$$

In the next section we describe the results [7, 10] of a semiclassical expansion of this effective string theory .

\section{The Semiclassical Calculation in the Background of a Rotating String}

We calculate $W[\Gamma]$ in the leading semiclassical approximation in the background of a worldsheet generated by a straight string attached to quarks rotating with uniform angular velocity $\omega$ (See Fig. 1).

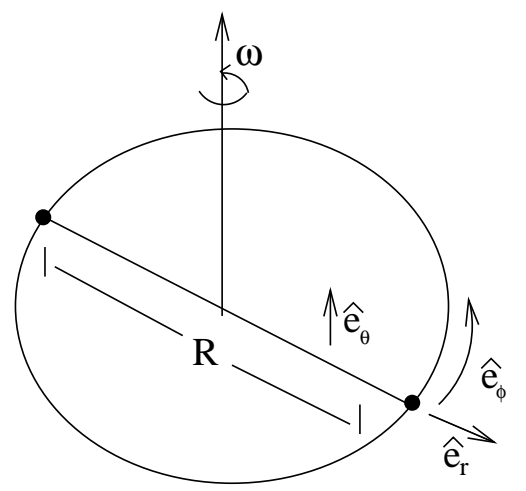

Figure 1. The string coordinate system

The quarks have masses $m_{1}$ and $m_{2}$, move with velocities $v_{1}=\omega R_{1}$ and $v_{2}=\omega R_{2}$, and are separated by a fixed distance $R=R_{1}+R_{2}$. The 
parameters $\xi=(t, r)$ are the time $t$ and the coordinate $r$, which runs along the string from $-R_{1}$ to $R_{2}$, so that the transverse velocity of the straight string is zero when $r=0$.

The amplitudes $f^{1}(\xi)$ and $f^{2}(\xi)$ of the transverse fluctuations are the spherical coordinates $\theta(r, t)$ and $\phi(r, t)$ of a point on the string. These angles are defined in an unconventional manner so that $\theta(r, t)=\phi(r, t)=0$ is a straight string rotating in the $x y$ plane. The angle $\theta(r, t)$ is the fluctuation perpendicular to the plane of rotation, and the angle $\phi(r, t)$ is the fluctuation lying in the plane of the rotating string.

The ends of the string are fixed to their classical trajectories,

$$
\theta\left(-R_{1}, t\right)=\theta\left(R_{2}, t\right)=\phi\left(-R_{1}, t\right)=\phi\left(R_{2}, t\right)=0 .
$$

The fluctuating worldsheet $\tilde{x}^{\mu}$ then has the parameterization $\tilde{x}^{\mu}(r, t)$ given by

$$
\begin{aligned}
\tilde{x}^{\mu}(r, t)= & x^{\mu}(\theta(r, t), \phi(r, t), r, t) \\
= & t \hat{\mathbf{e}}_{0}^{\mu}+r\left[\cos \theta(r, t) \cos (\phi(r, t)+\omega t) \hat{\mathbf{e}}_{1}^{\mu}\right. \\
& \left.+\cos \theta(r, t) \sin (\phi(r, t)+\omega t) \hat{\mathbf{e}}_{2}^{\mu}-\sin \theta(r, t) \hat{\mathbf{e}}_{3}^{\mu}\right],
\end{aligned}
$$

where $\hat{\mathbf{e}}_{\alpha}^{\mu}, \alpha=0, \ldots 3$ are unit vectors along the four fixed spacetime axes, $\hat{\mathbf{e}}_{\alpha}^{\mu}=\delta_{\alpha}^{\mu}$. The parameterization (12) is a concrete example of (3).

The classical rotating straight string $\bar{x}^{\mu}(r, t)$ has the parameterization,

$$
\begin{aligned}
\bar{x}^{\mu}(r, t) & =x^{\mu}(\theta(r, t)=0, \phi(r, t)=0, r, t) \\
& =t \hat{\mathbf{e}}_{0}^{\mu}+r\left[\cos \omega t \hat{\mathbf{e}}_{1}^{\mu}+\sin \omega t \hat{\mathbf{e}}_{2}^{\mu}\right] .
\end{aligned}
$$

The corresponding metric $\bar{g}_{a b}=g_{a b}\left[\bar{x}^{\mu}\right]$ and classical action $S_{\mathrm{NG}}\left[\bar{x}^{\mu}\right]$ are independent of the time $t$, so that $W[\Gamma]$ has the form

$$
W[\Gamma]=e^{i T L^{\text {string }}\left(R_{1}, R_{2}, \omega\right)},
$$

where $T$ is the elapsed time. For massless quarks, the ends of the string move with the velocity of light, and singularities appear in $L^{\text {string. To }}$ regulate these singularities, we retain the quark mass as a cutoff and take the massless limit at the end when evaluating physical quantities.

The Lagrangian $L^{\text {string }}$ is the sum of a classical part $L_{\mathrm{cl}}^{\text {string }}$ and a fluctuating part $L_{\text {fluc }}^{\text {string }}$,

$$
L^{\text {string }}=L_{\mathrm{cl}}^{\text {string }}+L_{\text {fluc }}^{\text {string }},
$$

where

$$
L_{\mathrm{cl}}^{\text {string }}=-\frac{\sigma}{T} \int d^{2} \xi \sqrt{-\bar{g}}=-\sigma \int_{-R_{1}}^{R_{2}} d r \sqrt{1-r^{2} \omega^{2}}
$$


We will calculate $L_{\text {fluc }}^{\text {string }}$ in $D$ spacetime dimensions. The fluctuation $\theta(r, t)$ is replaced by $D-3$ fluctuations perpendicular to the plane of rotation and there is still just 1 fluctuation in the plane of rotation.

The effective Lagrangian for the quark-antiquark pair is obtained by adding quark mass terms to $L^{\text {string: }}$

$$
L_{\mathrm{eff}}\left(R_{1}, R_{2}, \omega\right)=-\sum_{i=1}^{2} m_{i} \sqrt{1-v_{i}^{2}}+L^{\mathrm{string}}\left(R_{1}, R_{2}, \omega\right)
$$

The effective Lagrangian is the sum of a classical part and a fluctuating part,

$$
L_{\mathrm{eff}}\left(R_{1}, R_{2}, \omega\right)=L_{\mathrm{cl}}\left(R_{1}, R_{2}, \omega\right)+L_{\text {fluc }}^{\text {string }}\left(R_{1}, R_{2}, \omega\right)
$$

where

$$
\begin{aligned}
L_{\mathrm{cl}} & =-\sum_{i=1}^{2} m_{i} \sqrt{1-v_{i}^{2}}-\sigma \int_{-R_{1}}^{R_{2}} d r \sqrt{1-r^{2} \omega^{2}} \\
& =-\sum_{i=1}^{2}\left[m_{i} \gamma_{i}^{-1}+\sigma \frac{R_{i}}{2}\left(\frac{\arcsin \left(v_{i}\right)}{v_{i}}+\gamma_{i}^{-1}\right)\right],
\end{aligned}
$$

with

$$
\gamma_{i}^{-1}=\sqrt{1-v_{i}^{2}}, \quad v_{i}=\omega R_{i}
$$

The expression for $L_{\text {fluc }}^{\text {string }}$ is obtained from (14) and the semiclassical calculation of $W[\Gamma]$. It contains terms which are quadratically, linearly, and logarithmically divergent in the cutoff $M$. The quadratically divergent term is a renormalization of the string tension, the linearly divergent term is a renormalization of the quark mass, and the logarithmically divergent term is proportional to the integral of the scalar curvature over the whole worldsheet [11]. After absorbing the quadratically and linearly divergent terms into renormalizations, we obtain [7, 10]

$$
L_{\text {fluc }}^{\text {string }}\left(R_{1}, R_{2}, \omega\right)=\frac{\pi(D-2)}{24 R_{p}}-\sum_{i=1}^{2} \frac{\omega v_{i} \gamma_{i}}{\pi}\left[\ln \left(\frac{M R_{i}}{\gamma_{i}^{2}-1}\right)+1\right]+\frac{\omega}{2}+\omega f\left(v_{1}, v_{2}\right),
$$

where $R_{p}$ is the proper length of the string,

$$
R_{p}=\frac{1}{\omega}\left(\arcsin v_{1}+\arcsin v_{2}\right)
$$

and

$$
f\left(v_{1}, v_{2}\right)=-\frac{1}{\pi} \int_{0}^{\infty} d s \ln \left[\frac{s^{2}+\left(v_{1} \gamma_{1}+v_{2} \gamma_{2}\right) s \operatorname{coth}\left(s R_{p} \omega\right)+v_{1} \gamma_{1} v_{2} \gamma_{2}}{\left(s+v_{1} \gamma_{1}\right)\left(s+v_{2} \gamma_{2}\right)}\right] .
$$


The function $f\left(v_{1}, v_{2}\right)$ vanishes when $v_{1}$ and $v_{2}$ approach unity, so that the last term in (21) is small for relativistic quarks. The contribution to $L_{\text {fluc }}^{\text {string }}$ of the additional $D-4$ fluctuations perpendicular to the plane of rotation, coming from the extension to D dimensional spacetime, is contained in the first term in (21).

In the limit $\omega \rightarrow 0, R_{p} \rightarrow R_{1}+R_{2}=R$, and $L_{\text {fluc }}^{\text {string }}$ reduces to the result of Lüscher 12 for the correction to the static quark-antiquark potential due to string fluctuations,

$$
V_{\text {Lüscher }}=-L_{\text {fluc }}^{\text {string }}\left(R_{1}, R_{2}, \omega=0\right)=-\frac{\pi(D-2)}{24 R} .
$$

For $\omega \neq 0, L_{\text {fluc }}^{\text {string }}$ contains a logarithmically divergent part. We simplify this term using the classical equation of motion,

$$
\left.\frac{\partial L_{\mathrm{cl}}}{\partial R_{i}}\right|_{R_{i}=\bar{R}_{i}}=0
$$

to express $\bar{R}_{i}$ in terms of $\omega$. Eq. (25) gives the relation

$$
\sigma \bar{R}_{i}=m_{i}\left(\bar{\gamma}_{i}^{2}-1\right)
$$

where $\bar{\gamma}_{i}$ is equal to $\gamma_{i}$ evaluated at $R_{i}=\bar{R}_{i}$. The solution of (26) for $\bar{R}_{i}$ as a function of $\omega$ is

$$
\bar{R}_{i}=\frac{1}{\omega}\left(\sqrt{\left(\frac{m_{i} \omega}{2 \sigma}\right)^{2}+1}-\frac{m_{i} \omega}{2 \sigma}\right) .
$$

Using the relation (26) in (21) gives

$$
L_{\text {fluc }}^{\text {string }}(\omega)=\frac{\pi(D-2)}{24 R_{p}}-\sum_{i=1}^{2} \frac{\omega v_{i} \bar{\gamma}_{i}}{\pi}\left[\ln \left(\frac{M m_{i}}{\sigma}\right)+1\right]+\frac{\omega}{2}+\omega f\left(v_{1}, v_{2}\right) .
$$

Use of the classical equations of motion has eliminated the logarithmic dependence of $L_{\text {fluc }}^{\text {string }}$ on the dynamical parameter $\omega$.

The logarithmically divergent term in (28) is proportional to the quantity $\omega v_{i} \bar{\gamma}_{i}$ which diverges when $m_{i} \rightarrow 0$. This term can be absorbed into a renormalization of a term in the boundary action called the geodesic curvature [13] so that the theory will be finite in the $m_{i} \rightarrow 0$ limit.

\section{Renormalization of the Geodesic Curvature}

For a straight string rotating with angular velocity $\omega$, the geodesic curvature is equal to $\omega v_{i} \gamma_{i}^{2}$. The logarithmic divergence in (28) can then be 
removed by adding to the quark mass term in the boundary Lagrangian a counterterm containing the geodesic curvature. This gives

$$
L^{\text {boundary }}=-\sum_{i=1}^{2} \gamma_{i}^{-1}\left[m_{i}+\kappa_{i} \omega v_{i} \gamma_{i}^{2}\right],
$$

where the second term in $(29)$ is the geodesic curvature term. The logarithmic divergence in (28) can then be regarded as a renormalization of the coefficient $\kappa_{i}$ of the geodesic curvature in (29).

Since the quantity $\omega v_{i} \bar{\gamma}_{i}$ diverges when $m_{i} \rightarrow 0$, the requirement that the theory is finite in the $m_{i} \rightarrow 0$ limit forces the renormalized value of $\kappa_{i}$ to be zero. (We take the $m_{i} \rightarrow 0$ limit with the cutoff $M$ fixed, because we have an effective theory). Removing the terms in (28) proportional to $\omega v_{i} \gamma_{i}$ gives an expression for $L_{\text {fluc }}^{\text {string }}$ which is applicable in the massless quark limit,

$$
L_{\text {fluc }}^{\text {string }}=\frac{\pi(D-2)}{24 R_{p}}+\frac{\omega}{2}+\omega f\left(v_{1}, v_{2}\right) .
$$

In the case of two light quarks, $m_{1}=m_{2}=0\left(\bar{\gamma}_{1}, \bar{\gamma}_{2} \rightarrow \infty\right)$, Eqs. (22) and (23) give $R_{p}=\pi / \omega$ and $f\left(v_{1}, v_{2}\right)=0$, so that (30) becomes

$$
\left.L_{\text {fluc }}^{\text {string }}(\omega)\right|_{m_{1}=m_{2}=0}=\frac{\omega(D-2)}{24}+\frac{\omega}{2} .
$$

The first term in (31) is the negative of Lüscher potential with the length $R$ of the string replaced by its proper length $R_{p}=\pi / \omega$. It is the contribution of $D-2$ tranverse fluctuations in the background of a flat metric. The $\frac{\omega}{2}$ term accounts for the curvature of the classical background metric $\bar{g}_{a b}=g_{a b}\left[\bar{x}^{\mu}\right]$ generated by the rotating string.

In the case of one heavy and one light quark, $m_{1} \rightarrow \infty\left(v_{1} \rightarrow 0\right)$ and $m_{2}=0\left(\bar{\gamma}_{2} \rightarrow \infty\right), R_{p}=\pi / 2 \omega$ and

$$
f\left(v_{1}=0, v_{2} \rightarrow 1\right)=-\frac{1}{\pi} \int_{0}^{\infty} \ln \operatorname{coth}\left(\frac{\pi}{2} s\right)=-\frac{\omega}{4},
$$

so

$$
\left.L_{\text {fluc }}^{\text {string }}(\omega)\right|_{\substack{m_{1} \rightarrow \infty \\ m_{2}=0}}=\frac{\omega(D-2)}{12}+\frac{\omega}{4} .
$$

In this talk we use (31) to determine Regge trajectories of mesons composed of two light quarks. 


\section{Fluctuations in the Motion of the Quarks at the Ends of the String}

In the previous discussion, the quark-antiquark pair moved in a fixed classical trajectory in the $x y$ plane (See Fig. 1 and Eq's (11) and (27)). To take into account the fluctuations of the positions $\overrightarrow{\mathbf{x}}_{1}(t)$ and $\overrightarrow{\mathbf{x}}_{2}(t)$ of the quarks at the ends of the rotating string, we extend the functional integral (10) to include a path integral over $\overrightarrow{\mathbf{x}}_{1}(t)$ and $\overrightarrow{\mathbf{x}}_{2}(t)$, and add the action of the quarks to the string action (9). This extension replaces $W[\Gamma]$ by the partition function $Z$,

$$
Z=\frac{1}{Z_{b}} \int \mathcal{D} f^{1}(\xi) \mathcal{D} f^{2}(\xi) \mathcal{D} \overrightarrow{\mathbf{x}}_{1}(t) \mathcal{D} \overrightarrow{\mathbf{x}}_{2}(t) \Delta_{F P} e^{-i \sigma \int d^{2} \xi \sqrt{-g}+i S_{\text {quark }}},
$$

where $S_{\text {quark }}$ is the action of two free (scalar) quarks,

$$
S_{\text {quark }}=-\sum_{i=1}^{2} m_{i} \int_{-T / 2}^{T / 2} d t \sqrt{1-\dot{\vec{x}}_{i}^{2}(t)},
$$

and where $Z_{b}$ is the corresponding quark partition function ,

$$
Z_{b}=\int \mathcal{D} \overrightarrow{\mathbf{x}}_{1}(t) \mathcal{D} \overrightarrow{\mathbf{x}}_{2}(t) e^{i S_{\text {quark }}}
$$

Dividing by $Z_{b}$ removes the vacuum energy of the quarks.

We use the methods of Dashen, Hasslacher, Neveu [14 to carry out a semiclassical calculation of the partition function $Z$ around periodic classical solutions and determine the energies of the physical meson states. We find :

1. For massless quarks, the contribution to $L_{\text {eff }}(\omega)$ arising from the fluctuations in the motion of the ends of the string along the vector $\hat{e}_{r}$ shown in Fig. 1 vanishes. For massless quarks the longitudinal mode is unphysical and the meson energy comes from the fluctuations of the interior of the string, i.e. $L_{\text {fluc }}(\omega)=L_{\text {fluc }}^{\text {string }}(\omega)$.

(For massive quarks the fluctuations of the boundary also contribute to the meson energy.)

2. The usual WKB quantization condition for angular momentum.

The angular momentum $J$,

$$
J=\frac{d L_{\mathrm{eff}}(\omega)}{d \omega},
$$

takes on the values,

$$
J=l+\frac{1}{2} \quad l=0,1,2, \ldots
$$


The energy $E(\omega)$ is given by the corresponding Hamiltonian,

$$
E(\omega)=\omega \frac{d L_{\mathrm{eff}}(\omega)}{d \omega}-L_{\mathrm{eff}}(\omega)
$$

Eq's (19) and (31) give $L_{\text {fluc }}(\omega) / L_{\mathrm{cl}}(\omega) \sim \omega^{2} / \sigma \sim 1 / J$ so that for large $J, L_{\text {fluc }}(\omega)$ can be treated as a perturbation. The meson energy is then

$$
E(\omega)=E_{\mathrm{cl}}(\omega)-L_{\mathrm{fluc}}(\omega)
$$

where

$$
E_{\mathrm{cl}}(\omega)=\omega \frac{d L_{\mathrm{cl}}(\omega)}{d \omega}-L_{\mathrm{cl}}(\omega)
$$

and where $\omega$ is given as a function of $J$ by the classical relation

$$
J=\frac{d L_{\mathrm{cl}}(\omega)}{d \bar{\omega}} .
$$

Eqs. (42) and (40) determine meson Regge trajectories in the leading semiclassical approximation.

\section{Meson Regge Trajectories}

We first evaluate $L_{\mathrm{cl}}(\omega)$ to obtain classical Regge trajectories. For massless quarks Eqs. (19), (41), and (42) give

$$
L_{\mathrm{cl}}(\omega)=-\frac{\pi \sigma}{2 \omega}, \quad J=\frac{\pi \sigma}{2 \omega^{2}}, \quad E_{\mathrm{cl}}=\frac{\pi \sigma}{\omega}, \quad J=\frac{E_{\mathrm{cl}}^{2}}{2 \pi \sigma} .
$$

Using Eqs. (40), (31), and (43) to include the correction to the energy due to the zero point energy of the string fluctuations gives

$$
E(\omega)=E_{c l}(\omega)-L_{\text {fluc }}(\omega)=\frac{\pi \sigma}{\omega}-\frac{D-2}{24} \omega-\frac{\omega}{2} .
$$

The energies $E_{n}(\omega)$ of the excited states of the rotating string (light hybrid mesons) are obtained by adding to (44) the energies $k \omega$ of the excited

vibrational modes.

$$
E_{n}(\omega)=\frac{\pi \sigma}{\omega}-\frac{D-2}{24} \omega-\frac{\omega}{2}+n \omega .
$$

Since there are many combinations of string normal modes which give the same $n$ (e.g., a doubly excited $k=1$ mode and a singly excited $k=2$ mode each give $n=2$ ), the spectrum is highly degenerate. There are $D-2$ trajectories having $n=1$, each corresponding to a single excitation of one of the $k=1$ normal modes. Higher values of $n$ have higher degeneracies. 
The value of $\omega$ is given as a function of $J$ through the classical relation $\omega=\sqrt{\pi \sigma / 2 J}$. Squaring both sides of (45) yields

$$
\begin{aligned}
E_{n}^{2} & =2 \pi \sigma\left(J-\frac{D-2}{24}-\frac{1}{2}+n+O\left(\frac{n^{2}}{J}\right)\right) \\
& =2 \pi \sigma\left(l+n-\frac{D-2}{24}+O\left(\frac{n^{2}}{l}\right)\right),
\end{aligned}
$$

where we have used the WKB quantization condition (38). Setting $D=4$ in (46) and solving for $l$ we obtain the the Regge trajectories,

$$
l=\frac{E^{2}}{2 \pi \sigma}+\frac{1}{12}-n \quad n=0,1,2, \ldots
$$

The excited states, $n>0$, of the rotating string give rise to daughter Regge trajetories determining the spectrum of hybrid mesons composed of zero mass quarks. The first semiclassical correction to the leading Regge trajectory, $n=0$, adds the constant $1 / 12$ to the classical Regge formula. The small size of this correction could explain why Regge trajectories are linear at values of $l$ of order 1 .

\section{Comparison with Bosonic String Theory}

For $D=26$, Eq. (46) yields the spectrum

$$
E^{2}=2 \pi \sigma\left(l+n-1+O\left(n^{2} / l\right)\right) .
$$

The spectrum of energies (48) coincides with the spectrum of open strings in classical bosonic string theory. However, (48) is valid only in the leading semiclassical approximation, so that it cannot be used for $l=0$, where it would yield the scalar tachyon of the open bosonic string.

\section{Summary and Conclusions}

1. We have seen how the dual superconducting model of confinement leads to an effective string theory of long distance QCD. We have calculated, in the semiclassical large angular momentum domain, where this theory is applicable, the effect of string fluctuations on Regge trajectories for mesons containing light quarks. Similar calculations [10] give Regge trajectories for mesons containing one heavy and one light quark.

2. The spectrum of the energies of the excited states formally coincides with the spectrum of the open string of Bosonic string theory in its critical dimension $D=26$. Here, we obtained the spectrum for any $D$, 
but only for large $l$, from the semiclassical expansion of an effective string theory. The presence of functional determinant $\Delta_{F P}$ in the path integral (10) was essential in obtaining this result.

3. We treated the light quarks as massless scalar particles to determinine the energies of the high angular momentum excited states of mesons. However, the effect of chiral symmetry breaking, generating a constituent quark mass, must play a dominant role in determining the masses of mesons which are ground states of quark-antiquark systems.

4. The derivation of the effective string theory made no use of the details of the effective field theory from which it was obtained. Furthermore, arguments based on the work of 't Hooft [15] indicate that the confined phase of a non Abelian gauge theory is characterized by a dual order parameter, which vanishes in regions of space where "dual supercondutivity" is destroyed. This generic description of the confined phase of QCD leads to the effective string theory of long distance QCD described here, which provides a concrete picture of the QCD string.

\section{Acknowledgements}

We would like to thank S.Olejnik and the other organizers for providing the opportunity to participate in this stimulating workshop.

\section{References}

1. Y. Nambu, Phys. Rev. D10, 4262 (1974).

2. S. Mandelstam, Phys. Rep. 23C, 245 (1976).

3. G. 't Hooft, in High Energy Physics, Proceedings of the European Physical Society Conference, Palermo, 1975, ed. A. Zichichi (Editrice Compositori, Bologna, 1976).

4. H. B. Nielsen and P. Olesen, Nucl. Phys. B61, 45 (1973).

5. M. Baker, J. S. Ball and F. Zachariasen, Phys. Rev. D44, 3328 (1991).

6. M. Baker, J. S. Ball, N. Brambilla, G. M. Prosperi and F. Zacheriasen, Phys. Rev. D54, 2829 (1996).

7. M. Baker and R. Steinke, Phys. Rev. D69, 094013 (2001), hep-ph/0006069.

8. E. T. Akhmedov, M. N. Chernodub, M. I. Polikarpov and M. A. Zubkov, Phys. Rev. D53, 2087 (1996).

9. A. M. Polyakov, Gauge Fields and Strings, 151-191, (Harwood Academic Publishers, Chur, Switzerland, 1987).

10. M. Baker and R. Steinke, hep-th/0201169, to be published, Phys. Rev. D, (2002).

11. M. Lüscher, K. Symanzik and P. Weisz, Nucl. Phys. B173, 356 (1980).

12. M. Lüscher, Nucl. Phys B180, 317 (1981).

13. O. Alvarez, Nucl. Phys B216, 125 (1983).

14. R. Dashen, B. Hasslacher and A. Neveu, Phys. Rev. D10, 4114 (1974). (Cambridge University Press, 1987).

15. G.'t Hooft Nucl. Phys. B153, 141 (1979). 